\title{
Decision models in designing flexible production systems
}

\author{
Adriana Florescu ${ }^{1, *}$, and Sorin Adrian Barabaş ${ }^{1}$ \\ ${ }^{I}$ Transilvania University of Brasov, Faculty of Technological Engineering and Industrial \\ Management, Romania
}

\begin{abstract}
Flexible production system is a complex whole that raise some issues in terms of its design and in relation to the conditions for implementing it. To implement a flexible production system configuration must be found that satisfies both economic and system performance requirements. The configuration which best meet the objectives of introducing a flexible production system must be sought in the set of alternatives defined and evaluated. In this paper we present a methodology of realising the configuration and complex evaluation of the analyzed system. It will be developed models which generate new alternative configurations, optimization and evaluation models of the performance of the flexible production system. This will create a framework for interactive decision support, user-oriented that can be used by management to solve this selection problem. The applicative character of the study consist in tracking of the technological process in real time using the developed software package on the designed system, based on mathematical models for configuration and optimization of the system.
\end{abstract}

\section{Introduction}

The researches performed within the scientific paper, will be directed to the study of Flexible Manufacturing Systems (FMS), in order to know their behaviour and their performances very well, and if it is possible, before their physical manufacturing, and in order to establish on scientific bases dimensioning models, representation and simulation of FMS.

By introducing flexible production systems there can be obtained important advantages concerning the functioning of the organization and other economic advantages concerning: the possibility to change the reference (a new system adaptation) almost without losing time, for new equipment and disconnecting the attendance staff reported to the work pace of the machine, lead to an important increase of the use degree of the machine; fabrication in batches sizes, according to the necessary amount and at the time required by mounting (the possibility to deliver the pieces manufactured "Just in Time"), make possible an important decrease of the pieces volume in the workshop and warehouses (production decrease in processing and stocks, with a rate of 50-60\%) and it has influence upon a quick reaction capacity towards the market requirements oscillations, at the reference change;

* Corresponding author: fota.a@unitbv.ro 
production time decrease, often with more than $50 \%$, leads to delivery time decrease and thus to creating decisive competition advantages; improving quality by strictly respecting the stages of the production process; realizing a production process easily to be controlled in its essential points; work force qualification increase by its movement towards preponderantly intellectual activities; better use of the capital by its distribution between the buildings and the equipment of the process.

By the possibility of the optimal use both of the system capacity and the staff and their high availability, which can be reached today, of machines and plants, on the one hand and of almost unlimited enlargement, replacement and change flexibility, on the other hand, result important productivity increase on long terms of systems use (8-10 times reported to classical systems).

Taking into account the actual stage of the research and technical scientific achievements in the domain of flexible systems the following conclusions can be stated. Speciality literature $[1,2]$ offers disparate information concerning the construction and the implementing of flexible production systems:

- In their evolution of about three decades, there can be noticed efficient flexible systems implementing for cutting processes, but also many failures, determined most of the times by an over evaluation of technical solutions, by the impossibility of managing efficiently these systems which are extremely complex, by huge investment that they need. It is very difficult a correct substantiation of the investment costs, which most of the times exceed the expectancies;

- A difficulty which tempers the rhythm of FMS implementing is caused by the impact generated on the work market. The decrease sometimes dramatically of the work force necessary number, simultaneously with a qualification rise, by its movement towards intense intellectual activities, with a lot of stress, generates social problems and integration problems in the new environment;

- For now there isn't a unitary international definition for flexible manufacturing systems. The definition of the FMS concept differs from a country to the other and from an investigator to the other too. After gaining experience in their design and in their use, there was noticed a clear tendency to get different notions close to each other, as well as definitions on which the concept of flexible production systems are based;

- In most of the speciality works "flexibility" is often used as a "generic collocation" and it is not used in general, as a complex concept. Thus, in a very huge number of works „flexibility” is treated partially, superficially, in a manner which is confuse and sometimes wrong. There still are many opinions concerning the quantification way of the system flexibility. It is difficult to anticipate, in the concept stage, the optimal flexibility degree, as this is one of the major causes of the inefficiency concerning FMS exploitation. In most of the speciality works there is no distinction between the automation concept and the flexibility one. In reality they are different concepts;

- Actual flexible production systems are not always approached globally, interdisciplinary. Due to their complexity, these systems need a synthesis approach and implicitly the adequate expert. There is not presented a clear methodology, accepted by everybody in order to determine the structure of the flexible production systems, according to the system's inputs and outputs and to the interdependences between them and the exterior environment.

Advanced production systems development requires profound and continues changes which will affect both the technical structure, through increased automation, robotics and information technology, and also the methods and techniques of management and organization of such systems. To ensure their competitiveness, companies will have to rethink a new strategy design and implementation of these new and efficient systems. 


\section{Modeling in Flexible Production Systems}

As far as the functioning modelling of the flexible production systems is concerned, there still are a lot of attempts to define and analyse the performance indicators of the system [3]. From the point of view of global characteristics, flexible production systems are superior to production systems which they replace. The huge number of the diverse configurations of flexible production systems showed that it is possible the flexible automation in the domain of individual fabrication, of small series and medium one, both from technical and economic point of view.

\subsection{Models applied in the design of FMS}

The decision models encountered in the literature [4, 5] that allow selecting optimal alternative configuration of a flexible production system have different characteristics depending on conditions requirements for sizing and configuration of FMS. When designing any flexible manufacturing system it is taken into account the following: the behaviour in time of FMS, degree of flexibility, decision variables of FMS, certainty of data programming / planning.

The behaviour in time of a FMS is analyzed by applying two types of models: dynamic and static model. Dynamic models are applied generally in process modeling of waiting at the workstation in the system, traceability of materials and flexible manufacturing system simulation as a large, complex dynamic system. Thus, basic models come from queuing theory and dynamic of large systems. Static models include in the analysis i.e. calculation of productivity of the workstation. Productivity of the entire flexible manufacturing system is determined by the productivity of the "bottleneck" workstation. Linear programming models are used.

The degree of the flexibility of the system requires the use of the following two types of decision models: models with one period, where the analysis focuses on the representative period for which it can be designed a acceptable configuration of FMS; multi-period models used to establish a development strategy and step by step adjustment of the system configuration depending on the required production changes. The lifetime of FMS is divided into sub-periods, each with its own program of production.

Decision variables in a flexible production system are based on models that trace products association to routes and operations to machines and models for selecting the equipment that will be integrated into FMS. The models which aim associating products at routes and operations to machines are used to determine the sequence of operations (technological plan itineraries / routes) consistent with planned production. The models that allow the selection of equipment to be integrated in FMS, allow the determination of different equipment: machines, working posts, workstations, loading / unloading station, industrial logistics / vehicles to be included in FMS. Models for determining the type and number of pallets or devices determine the right level of pallets. The number of pallets circulating in FMS determines how the available capacity is used.

The certainty degree of the planning data requires the use of deterministic and stochastic models. The deterministic models take into account that the information received from the outside about outputs performed in FMS are considered to be certain. The stochastic models are premised on the fact that the desired outputs are the result of a forecasting process which is, in many cases, the topic of greatest uncertainty.

Modelling and simulation of a flexible manufacturing system involves creating a model of the system and implement it in a programming language, aimed to checking the functioning of the system and assessing its performance. 


\subsection{A model for optimal configuration of a Flexible Manufacturing System}

On the basis of a flexible manufacturing system design should stand current task production, characterized by a predetermined typology, [6]. In order to choose the type and number of workstations in a flexible manufacturing system will be developed methodology for determining the degrees of consistency, affinity and differentiation and will make their quantitative analysis. As a result of this analysis, conclusions as follows are coming out, regarding time and working station number of flexible manufacturing systems selection as well as machine number of each working station. These conclusions were be used in this paper in view of drawing up the graphic model.

The affinity degree is determined by the equation (1):

$$
A D=m_{g}\left\{T O_{i}\left(I_{k}\right)\right\}, 1 \leq k \leq r, 1 \leq i \leq q
$$

where: $T O_{i}$ are the technological operations, $I_{k}$ - generalized item and $m_{g}$ - different commune elementary geometrical components of all generalized manufacturing task. This expresses reducing of total number of workstation $\left(q_{\text {total }}\right)$ of the FMS, increase of the processing process in flexible manufacturing system concentration degree, by the group technology.

FMS results as a less flexible structure, objective expected in design by: $\min q_{\text {total }}$ $\left\{m_{i}, 1 \leq i \leq q\right\} ; m_{i}$ - machines in system; $n_{g}$ - representing the total number of commune and not commune geometrical elements - in equation (2), which defines the generalized item $\left(I_{k}\right)$ and then, the typological nucleus of generalized manufacturing task; it results that difference expresses $\left(n_{g}-m_{g}\right)$ the not-commune number geometrical elements (completely different, specific) of different items included in manufacturing task, which distinguish them and technologic by different complementary technological operations $\left(T O_{c}\right)$ and additional technological operations $\left(T O_{a}\right)$. This difference expresses a certain indicator of the processing process division degree in flexible manufacturing system which can't be made by group technology, but only by different $T O_{i}\left(T O_{c}\right)$ technologies.

Consequently the difference (equation 2) - degree of differentiation (DD) needs just as much $\left(m_{i}\right)$ of different types:

$$
\begin{gathered}
D D=(C D-A D)=\left(n_{g}-m_{g}\right) T O_{c} \\
q_{\text {different }}=\left(n_{g}-m_{g}\right) T O_{i}
\end{gathered}
$$

The number $\left(m_{i}\right)$ on which the different $T O_{i}$ types for some specific item included in generalized manufacturing task are executed. But, min $m_{g} T O_{i}$ expresses on the one hand different $\left(m_{g}\right) T O_{i}$, representing commune $\left(m_{g}\right) T O_{c}$, consecutively executable by group technology. Consequently, flexible manufacturing systems should include:

$$
q_{\text {commune }}=\min m_{g}\left(T O_{i}\right)
$$

which will execute the commune geometrical elements $\left(m_{g}\right)$ and commune technological operations $\left(T O_{i}\right)$ for all items included in generalized manufacturing task.

From the technological point of view flexible manufacturing system is consisting of:

$$
q_{t o t}=q_{c o m}+q_{d i f}=\min m_{g}\left(T O_{i}\right)+\left(n_{g}-m_{g}\right)\left(T O_{i}\right)
$$


Table 1 presents the values of affinity degrees $(A D)$, consistency degrees $(C D)$ and the difference $(C D-A D)$, calculated (in \%) according to the previous methodology for designed FMS in which considered six family parts $\left(F P_{1 \ldots F P_{6}}\right),[6]$ :

$$
\begin{aligned}
& C D=\frac{n_{g k}}{n_{g 1}} \\
& A D=\frac{m_{g k}}{n_{g 1}} \\
& D D=\frac{n_{g k-} m_{g k}}{n_{g 1}}
\end{aligned}
$$

Table 1. Analysis of affinity, consistency and differentiation degrees

\begin{tabular}{|l|l|l|l|l|l|}
\hline$F P_{k}$ & $n_{\mathrm{gk}}$ & $\mathrm{m}_{\mathrm{gk}}$ & $\mathrm{CD}[\%]$ & $\mathrm{AD}[\%]$ & $\mathrm{DD}[\%]$ \\
\hline $\mathrm{FP}_{1}$ & 50 & 47 & 100 & 94 & 6 \\
\hline $\mathrm{FP}_{2}$ & 48 & 42 & 96 & 84 & 12 \\
\hline $\mathrm{FP}_{3}$ & 30 & 28 & 60 & 56 & 4 \\
\hline $\mathrm{FP}_{4}$ & 27 & 27 & 54 & 54 & 0 \\
\hline $\mathrm{FP}_{5}$ & 29 & 28 & 58 & 56 & 2 \\
\hline $\mathrm{FP}_{6}$ & 27 & 25 & 54 & 50 & 4 \\
\hline
\end{tabular}

Based on consistency degrees, affinity and differentiation degrees determined by the methodology developed it is presented in Figure 1 the general structure of FMS analyzed for the six families of cylindrical parts.

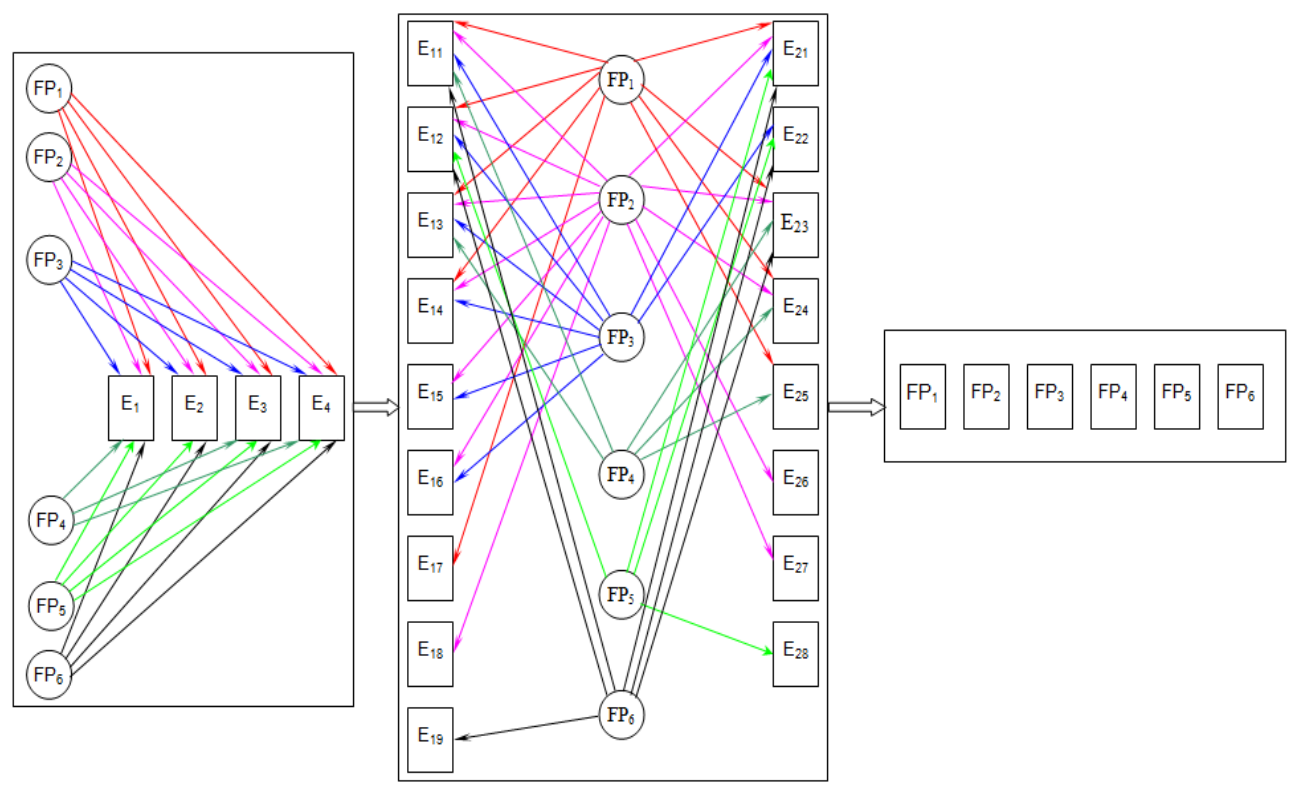

Fig. 1. The structural scheme of the system

Determining the number and type of machine-tools, technological equipment, workstations, stations of measuring - control and their arrangement in system was based on the following factors: total production volume expressed as number of items processed and total unit cost / piece, material flow, technological itineraries and other components of the FMS. 


\subsubsection{Establishing the optimal configuration and simulation of the system}

Configure a handling system depends mainly on: typological diversity of the task of production, the type of objects handled, the amount of products handled, transport distances, type of production system serviced, the pace imposed of the material flow, diagram routes material flows, routes transport. The basic configurations of a FMS commonly found $[1,6]$ are in a straight line (open), in the loop (closed), in large scale open expanses and centred on robot cells. A major influence on the determination of the structure of this FMS had the degree of affinity or differentiation. The basis for establishing the system configuration is the relationship systemic between information technological flow the technology of group on entry and task manufacturing generalized from out of the system, [6].

In Figure 2 is shown the system designed, on which was implemented and validate the optimization model previously developed. To study the behaviour of a real system for purposes of control and management of the system was developed a simulation program using the programming environment $\mathrm{Cx}$-Supervisor Developer.
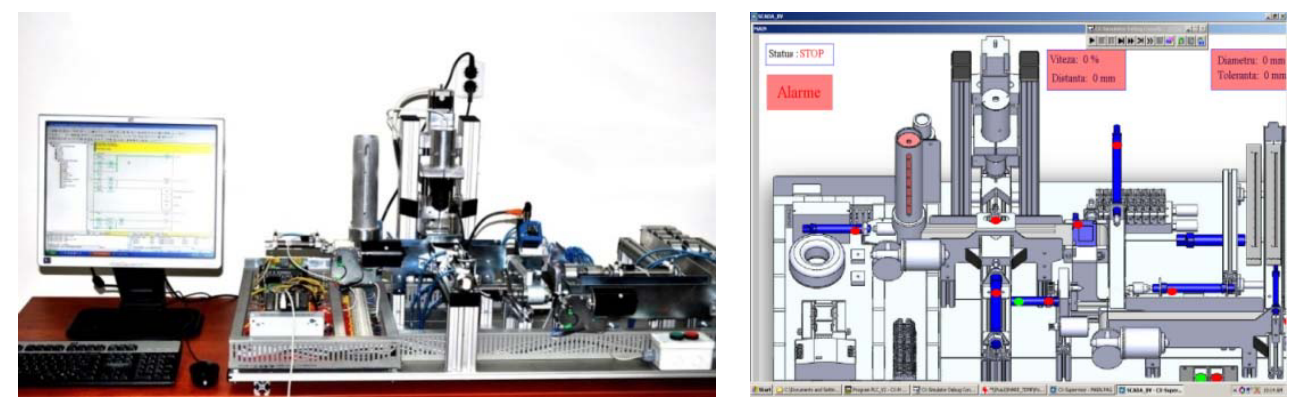

Fig. 2. The structuring and simulation of FMS. The sequence of the application - simulation software

This allows for modification of the technological flux based on relevant factors: number and type of workstations and tools-machines from the system, number and type of vehicles (conveyors) and speed of transport, logistics subsystem type used, the type of parts and their technological route, duration of the processing cycle of each type of piece to each module of the flexible system, the number of parts simultaneously in processing and batch to batch volume.

\section{The model of decision in route optimization}

Mathematical optimization models used in the configuration of flexible production systems aimed at reducing the number of different alternative configurations and thus to exclude those suboptimal configurations, in the previous stage of the system designing. This decision problem applies to complex systems there are different routes that can be used alternatively and / or in parallel for manufacturing a typological diversity of parts.

Different mathematical optimization approaches proposed in the literature [5 - 9], covering the wide range of different variables of decision. Decision-making process in determining the optimal alternative configuration of the FMS is to establish the first performance evaluation procedures that allow rapid a rough estimate of the performance of FMS. Then, the process continues progressively to leave some configurations that will be analyzed in detail. Thus, it is possible to eliminate a significance number of alternatives, earlier with a small computational effort. In general, the procedure is based on the classical model of closed queuing networks systems (the CQN model) [10]. 


\subsection{The optimization model}

The model of decision for route optimization associates the share of production for different types of items on different routes. To develop optimization model can be considered following inputs:

- are defined several routes of transport available $\left(\mathrm{R}_{\mathrm{k}}\right): i=\left(1, \ldots, R_{k}\right)$;

- the share of production is considered variable: $Q_{k i}=v a r$

The mathematical model of distribution of the total volume of production (total share of production $\left.-Q_{t k}\right)$ on alternative routes $\left(R_{k}\right)$ is shown in equation (1):

$$
Q_{t k}=\sum_{i=1}^{R_{k}} Q_{k i}, k=1, \ldots, n
$$

$Q_{k i}$ share of production is calculated based on the ratio of the average number of parts / items $\left(P_{k}\right)-k$ per unit time manufactured on processing routes $\left(R_{k}\right)$ and number of parts $-k$ manufactured per unit time. Thus, by variable $Q_{k i}$ are optimizing the most significant indicators of performance such as the productivity and waiting times in the system.

A frequent optimization model in the literature $[5,10]$ is the linear optimization model for determining the optimum distribution of the productivity of individual parts of transport routes.

It is considered that the system works with a large number of parts (default paddles) and the objective optimization is to maximize productivity by associating parts on various routes. It can be determined the degree of load $(w)$ in each workstation $(q)$ for each of the routes $(i)$, according to the equation (9), in which: $T O_{p q i}$ - average number of operations from workstation $(q)$ for parts $(k)$ processed on routes $(i) ; \bar{T}_{p q i}$ - average time for processing workpieces $(p)$ from the station $(q)$ moving on the route $(i)$.

$$
\begin{gathered}
w_{k q i}=T O_{k q i} \cdot \bar{T}_{k q i} \\
q=1,2, \ldots, m ; k=1,2, \ldots P_{k} ; i=1,2, \ldots, R_{k}
\end{gathered}
$$

The decision model is represented by the objective function (9). Decision variables [10] are the number of parts processed per unit of time and moving on the alternative routes $\left(x_{k i}\right)$.

$$
\max Q(x)=\sum_{k=1}^{P_{k}} \sum_{i=1}^{R_{k}} x_{k i}
$$

The objective function describes the maximize productivity of the entire flexible production system, which is the sum of the average number of parts that are processed per unit time in the system. The variables in the model are: $Q(x)$ - productivity of FMS and $x_{k i}$.

Are imposed the conditions, where $\mathrm{N}_{\mathrm{q}}$ is number of machines / workstation in system:

$$
\begin{array}{cc}
Q_{t k} \sum_{p=1}^{P_{k}} \sum_{i=1}^{R_{k}} x_{p k}=\sum_{i=1}^{R_{k}} x_{k i} & k=1,2, \ldots, P_{k} \\
\sum_{k=1}^{P_{k}} \sum_{i=1}^{R_{k}} x_{k i} \cdot w_{k q i} \leq N_{q} & q=1,2, \ldots, m
\end{array}
$$

The linear optimization model is developed and it can be solved with a standard algorithm. Some of the results obtained and validated on designed system are presented in Table 2.

The objective function from equation (9) expresses maximize productivity whole system and represents the average number of parts that are processed per time unit in the system. Productivity of the system includes the sum of all variables, according to equation (9). 
The share of production of each type of piece was obtained by dividing the parts productivity at the system productivity.

Table 2. Results. The optimal solution

\begin{tabular}{|l|l|l|l|l|}
\hline Part $P_{k}$ & Route $\mathrm{R}_{\mathrm{k}}$ & $\begin{array}{l}\text { Decision } \\
\text { variables }\end{array}$ & $Q_{k i}$ & $Q_{t k}$ \\
\hline$P 1$ & 1 & $x_{11}$ & 0,0934 & 0,18 \\
\hline$P 2$ & 2 & $x_{12}$ & 0,0866 & \\
\hline$P 3$ & 1 & $x_{21}$ & & 0,13 \\
\hline$P 4$ & 1 & $x_{31}$ & & 0,12 \\
\hline$P 5$ & 1 & $x_{41}$ & 0,0729 & 0,14 \\
\hline$P 6$ & 2 & $x_{42}$ & 0,0671 & 0,12 \\
\hline$P 7$ & 1 & $x_{51}$ & 0,0818 & 0,16 \\
\hline$P 8$ & 2 & $x_{52}$ & 0,0782 & \\
\hline$P 9$ & 1 & $x_{61}$ & & 0,15 \\
\hline
\end{tabular}

\section{Conclusions}

Based on research conducted were developed graphic-analytical dynamic model to configure the FMS and the optimization system model. A flexible system was designed and optimized, including industrial logistics subsystem and subsystem management process. Was performed simulation of functioning of the system and optimization in real-time environment and by implementing a monitoring software and SCADA control, using programming environment $\mathrm{Cx}$-Supervisor Developer.

The applicative character of the research theme is demonstrated simulating the flexible manufacturing system's performance for cylindrical parts processing. After simulating the functioning of the flexible production system, the validity of the models will be confirmed, as well as their utility in the design and the management of the processes in real time and environment. The implementation of flexible production system in an integrated production system, is an important result, which leads to optimization of the material flows and informational of enterprise.

\section{References}

1. M. P. Groover, Automation, Production Systems and Computer-Integrated Manufacturing, $3^{\text {rd }}$ Edition, Prentice Hall, Pearson (1987)

2. K. H. Han, Int. J. Math. Comp. Sim., 6, 4 (2012)

3. D. Catrina, Flexible Manufacturing Systems, MatrixRom Publish House, Bucharest (2008)

4. B.S.P. Reddy, C.S.P. Rao, Int. J. Sim. Mod., 10 (2011)

5. I. Bojan, Flexible Production Systems, Dacia Publish House, Cluj-Napoca (1999)

6. A. Fota, Designing of the machines systems. Modeling and simulation, Transilvania University of Brasov Publish House (2004)

7. M. Anderson, G. Olsson, Proceedings of 1998 Winter Simulation Conference, Washington, DC, pp. 935-941 (1998)

8. G. A. Chang, W. R. Peterson, 122 nd Anual Conference\& Exposition, June 14-17, Seatle, WA, American Society for Engineering Education (2015)

9. F. Zammori, M. Braglia, M. Frosolini, Int. J. Ind. Eng. Comp., 2, p. 593-616 (2011)

10. G. Secco-Suardo, Optimization of a Closed Network of Queues, Electronic Systems Laboratory, MIT, Cambridge, Massachusetts (1978) 